\title{
Modelo de Canal de Estados Finitos para Canais com Desvanecimento Correlacionado no Tempo e Decisão Suave
}

\author{
Igor Moreira e Cecilio Pimentel
}

\begin{abstract}
Resumo-Modelos de canais de estados finitos Markovianos (FSMC, do inglês Finite State Markov Channel) são comumente usados para caracterizar a memória de canais discretos binários (entrada binária, saída binária). Este trabalho propõe um novo modelo pertencente à classe FSMC, denominado de modelo com apagamento (MA), que foi idealizado para modelar canais discretos não binários (entrada binária, saída ternária) com memória. Os parâmetros do modelo MA serão estimados de modo a aproximar um canal discreto composto por um modulador BPSK, um canal com desvanecimento Rayleigh com função de autocorrelação com decaimento exponencial e ruído aditivo Gaussiano branco, um demodulador coerente e um quantizador com três níveis de quantização. Esta estimação será utilizada usandose o método da minimização da distância Kullback-Leibler. Para avaliar a exatidão do modelo proposto, serão comparadas as curvas da função autocorrelação obtidas analiticamente para o canal discreto e para o modelo MA.
\end{abstract}

Palavras-Chave-Canais de estados finitos, cadeias de Markov, desvanecimento plano, quantização.

Abstract-Finite-state Markov channels (FSMC) are commonly used to characterize the memory of binary (binary-input, binary-output) discrete channels. This work proposes a new FSMC model, called model with erasure (MA), to characterize non-binary (binary-input, ternary-output) discrete channels with memory. The MA is used to model a discrete communication system composed of a BPSK modulator, a channel with Rayleigh fading with a known autocorrelation function and additive white Gaussian noise, a coherent demodulator and a three-level quantizer. The parameters of the MA are found by minimizing the Kullback-Leibler distance. To evaluate the accuracy of the proposed model, the autocorrelation functions obtained analytically for the discrete channel and for the MA are compared.

Keywords-Finite state channel, Markov chain, flat fading, quantizer.

\section{INTRODUÇÃO}

Canais de comunicações sem fio estão sujeitos a uma série de distúrbios que variam no tempo, tais como, regiões de sombreamento e desvanecimento por multipercurso. Uma característica comumente presente nestes canais é que os erros ocorrem em surtos, indicando a existência de uma correlação no processo de geração de erros [1]. Devido à esta dependência estatística, o canal é dito ser um canal com memória.

Utiliza-se normalmente a técnica de entrelaçamento para eliminar ou diminuir os efeitos da memória do canal. Este

Igor Moreira, Agência Nacional de Telecomunicações, Brasília-DF, email: igormoura@anatel.gov.br. Cecilio Pimentel, CODEC/DES/UFPE, Recife-PE, Brazil, email: cecilio@ufpe.br.

Este trabalho recebeu suporte parcial do CNPq, no. 304173/2007-0. trabalho foi realizado partindo-se da premissa que a memória dos canais com desvanecimento não pode ser ignorada, pois é possível efetuar transmissões confiáveis utilizando-se estratégias de correção de erros projetadas de acordo com o comportamento da memória do canal [2], [3]. Além disso, a não utilização da memória do canal significa uma perda de capacidade [2].

Um canal de estados finitos Markoviano (FSMC, do inglês finite state Markov channel) é um canal discreto que possui um conjunto finito de estados, com transição de estados descrita por uma cadeia de Markov, a qual tem probabilidades de transição atribuídas de forma independente do tempo. A cada estado é associada uma determinada probabilidade de geração de erros. Modelos FSMC binários (entrada binária, saída binária) têm sido amplamente utilizados para descrever estruturas de correlação de erros em canais sem fio com memória [4]-[7]. Embora estes trabalhos sejam restritos à canais binários, estudos em teoria da informação revelam que a decisão suave pode aumentar significativamente a capacidade de várias classes de canais [8], [9].

Este trabalho propõe um modelo FSMC não binário com o intuito de capturar simultaneamente a memória do canal e a informação suave. O modelo proposto, denominado Modelo com Apagamento (MA), foi idealizado para modelar um sistema de comunicações composto por um modulador BPSK, um canal com desvanecimento Rayleigh correlacionado no tempo com função autocorrelação com decaimento exponencial, um demodulador coerente e um quantizador com três níveis de quantização. Esse sistema foi denominado canal discreto com correlação exponencial (CDCE). A introdução de um terceiro nível de quantização tem o propósito de indicar ao decodificador de canal que uma decisão sobre os bits transmitidos não foi realizada neste intervalo.

Uma vez definido o modelo MA, seus parâmetros serão estimados de modo a aproximá-lo do CDCE. Para isso, será utilizado um método de minimização da divergência, mensurada pela distância Kullback-Leibler [10]. Para avaliar a exatidão do modelo proposto, serão comparadas as curvas da função autocorrelação obtidas para o MA e as obtidas analiticamente para o CDCE. Este trabalho contribui com técnicas de modelamento e ferramentas para avaliação de desempenho de canais com memória.

\section{Modelo com Apagamento}

O modelo de canal com apagamento, denominado de MA, foi idealizado como um FSMC não-binário, com três níveis de 

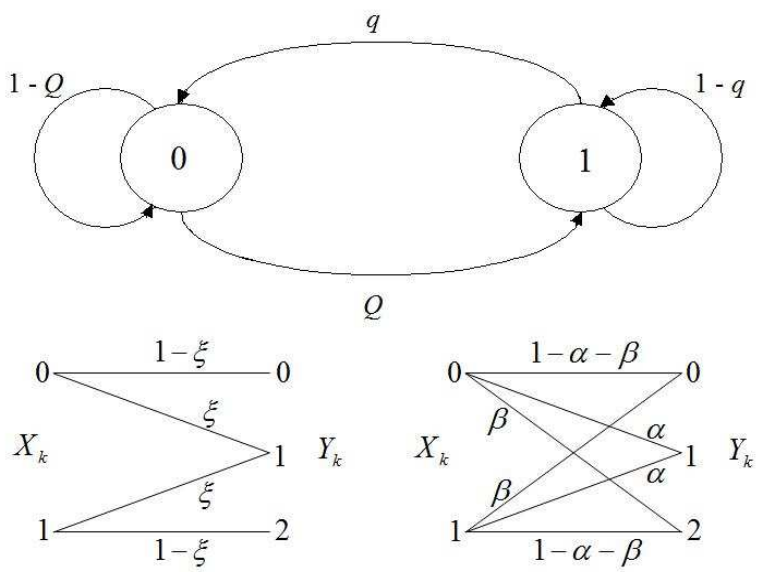

Fig. 1. Modelo com apagamento para canais com memória.

quantização. As seqüências de variáveis aleatórias na entrada e saída do canal são denotadas, respectivamente, por $\left\{X_{k}\right\}_{k=1}^{\infty}$ e $\left\{Y_{k}\right\}_{k=1}^{\infty}$, onde $X_{k} \in\{0,1\}$ e $Y_{k} \in\{0,1,2\}$. As distorções e interferências causadas pelo canal são representadas pela seqüência de ruído ternária $\left\{Z_{k}\right\}_{k=1}^{\infty}$, onde $Z_{k} \in\{0,1,2\}$. É dito que ocorreu um erro na recepção, no $k$-ésimo intervalo, se $Z_{k}=2$, um apagamento se $Z_{k}=1$, ou a recepção foi correta se $Z_{k}=0$.

O canal MA consiste de uma cadeia de Markov com dois estados, como ilustrado na Fig. 1. Quando a cadeia se encontra no estado 0 , ou estado bom, a probabilidade de erro é nula, a probabilidade de apagamento é $\xi$ e a probabilidade de um bit ser transmitido corretamente é igual a $1-\xi$. O processo de geração do apagamento associado ao estado 0 é representado por um canal binário com apagamento, BEC (do inglês Binary Erasure Channel). Esse estado representa a transmissão com boa qualidade, em que a probabilidade de erro é desprezível quando comparada com a probabilidade de acerto e de apagamento. Quando a cadeia se encontra no estado 1 (denominado de estado ruim), ocorrerá um erro com probabilidade $\beta$, um apagamento com probabilidade $\alpha$ e a probabilidade de acerto é igual a $1-\alpha-\beta$. A geração do dígito $z_{k}$ no estado 1 é representada por um canal discreto sem memória, DMC (do inglês Discrete Memoryless Channel). A probabilidade de transição do estado 0 para o estado 1 é dada por $Q$, e a probabilidade de transição do estado 1 para o estado 0 é dada por $q$.

É possível representar a probabilidade de ocorrer uma sequiência de ruído $\mathbf{z}_{n}=\left(z_{1} \ldots z_{n}\right)$, de comprimento $n$, em uma forma matricial. Para isso, seja $\mathbf{P}\left(z_{k}\right), z_{k} \in\{0,1,2\}$, uma matriz $2 \times 2$, cujo $(i, j)$-ésimo elemento é a probabilidade da cadeia transicionar do estado $i$ para o estado $j$ e gerar um dígito de ruído $z_{k}$. Então

$$
P\left(\mathbf{z}_{n}\right)=\boldsymbol{\Pi}^{T}\left(\prod_{k=1}^{n} \mathbf{P}\left(z_{k}\right)\right) \mathbf{1}
$$

onde $\boldsymbol{\Pi}^{T}=\left[\pi_{0}, \pi_{1}\right]$ é o vetor de probabilidade estacionária, na qual o sobrescrito [. $]^{T}$ indica a transposta da matriz, e 1 é um vetor coluna com todos os elementos iguais a um. $\mathrm{O}$ canal
MA é especificado pelas matrizes $\mathbf{P}(0), \mathbf{P}(1)$ e $\mathbf{P}(2)$, dadas, respectivamente, por:

$$
\begin{gathered}
\mathbf{P}(0)=\left[\begin{array}{cc}
(1-Q)(1-\xi) & Q(1-\alpha-\beta) \\
q(1-\xi) & (1-q)(1-\alpha-\beta)
\end{array}\right] \\
\mathbf{P}(1)=\left[\begin{array}{cc}
(1-Q) \xi & Q \alpha \\
q \xi & (1-q) \alpha
\end{array}\right] \\
\mathbf{P}(2)=\left[\begin{array}{cc}
0 & Q \beta \\
0 & (1-q) \beta
\end{array}\right] .
\end{gathered}
$$

A matriz de probabilidade de transição da cadeia de Markov é dada por $\mathbf{P}=\mathbf{P}(0)+\mathbf{P}(1)+\mathbf{P}(2)$ e o vetor de probabilidade estacionária dos estados é $\boldsymbol{\Pi}=[q /(Q+q), Q / Q+q]^{T}$.

É possível calcular a probabilidade de qualquer seqüência de erros, apagamentos e recepções corretas em regime estacionário utilizando (1). Seja $P_{\mathrm{MA}}(j) \triangleq P\left(Z_{k}=j\right)$, então

$$
\begin{aligned}
P_{\mathrm{MA}}(2) & =\frac{Q}{Q+q} \beta \\
P_{\mathrm{MA}}(1) & =\frac{q}{Q+q} \xi+\frac{Q}{Q+q} \alpha \\
P_{\mathrm{MA}}(0) & =\frac{q}{Q+q}(1-\xi)+\frac{Q}{Q+q}(1-\alpha-\beta) .
\end{aligned}
$$

A média do processo $\left\{Z_{k}\right\}_{k=0}^{\infty}$, denotada por $\mu$, é dada por $\mu=(Q(\alpha+2 \beta)+q \xi) /(Q+q)$. A variância, denotada por $\sigma^{2}$, é dada por:

$$
\sigma^{2}=\frac{Q(\alpha+4 \beta)+q \xi}{Q+q}-\left(\frac{Q}{Q+q}(\alpha+2 \beta)+\frac{q}{Q+q}(\xi)\right)^{2} .
$$

O coeficiente de correlação, denotado por $\operatorname{Cor}_{\mathrm{MA}}$, escreve-se:

$\operatorname{Cor}_{\mathrm{MA}}=\frac{(q+Q)\left[q Q\left(-2 \xi \alpha-4 \xi \beta+4 \alpha \beta+\xi^{2}+\alpha^{2}+4 \beta^{2}-4 \alpha^{2}\right)-4 Q \beta^{2}-q \xi^{2}-Q \alpha^{2}\right]}{q^{2}\left(-\xi+\xi^{2}\right)+q Q(-\xi-\alpha-4 \beta+2 \xi \alpha+4 \xi \beta)+Q^{2}\left(-\alpha-4 \beta+\alpha^{2}+4 \beta^{2}\right)}$.

\section{A. Função Autocorrelação}

A função autocorrelação do processo $\left\{Z_{k}\right\}_{k=0}^{\infty}, \operatorname{com} Z_{k} \in$ $\{0,1,2\}$, é dada por [11]:

$$
\begin{aligned}
R(k) & =\mathbf{E}\left\{Z_{i} Z_{i+k}\right\} \\
& = \begin{cases}\sum_{m} \sum_{n} m n P\left(Z_{i}=m, Z_{i+k}=n\right), & \text { se } k \neq 0 \\
\sigma^{2}+\mu^{2}, & \text { se } k=0\end{cases}
\end{aligned}
$$

em que $m, n \in\{0,1,2\}, \sigma^{2}$ é a variância do processo $\left\{Z_{k}\right\}_{k=0}^{\infty}$ e $\mu$ é a sua média. De acordo com [11], uma expressão matricial para a probabilidade $P\left(Z_{i}=m, Z_{i+k}=\right.$ $n), m, n \in\{0,1,2\}, k \neq 0$, de um FSMC em função das matrizes $\mathbf{P}(i), i \in\{0,1,2\}$, é dada por:

$$
P\left(Z_{i}=m, Z_{i+k}=n\right)=\boldsymbol{\Pi}^{T} \mathbf{P}(m) \mathbf{P}^{|k|-1} \mathbf{P}(n) \mathbf{1} .
$$

Para o modelo MA, substitui-se as matrizes $\mathbf{P}(0), \mathbf{P}(1)$ e $\mathbf{P}(2)$, dadas em (2)-(4) em (8) e, após algumas simplificações, chega-se à seguinte expressão:

$$
R(k)=\mu^{2}+\frac{q Q(\alpha+2 \beta-\xi)^{2}(1-q-Q)^{k}}{(Q+q)^{2}} .
$$

O modelo de um sistema de comunicações discreto que desejase modelar com o MA será descrito na próxima seção. 


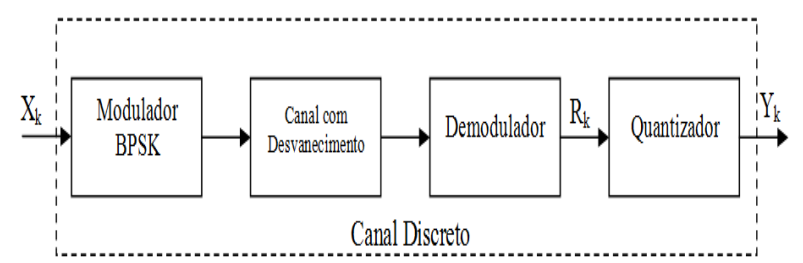

Fig. 2. Sistema de comunicações com modulador BPSK, canal com desvanecimento Rayleigh, demodulador e quantizador.

\section{Modelo do Sistema de Comunicações Discreto}

Considera-se um sistema de comunicações composto por um modulador BPSK, um canal com desvanecimento Rayleigh correlacionado no tempo e ruído aditivo gaussiano branco, um demodulador coerente e um quantizador com três níveis de quantização, como ilustrado na Fig. 2.

A envoltória complexa do desvanecimento $\widetilde{G}(t)=\widetilde{G}_{I}(t)+$ $\jmath \widetilde{G}_{Q}(t)$ é um processo Gaussiano complexo, estacionário no sentido amplo, com média zero, $\mathbf{E}[\widetilde{G}(t)]=0$ e segundo momento normalizado, $\mathbf{E}\left[|\widetilde{G}(t)|^{2}\right]=1$. Os componentes em quadratura $\widetilde{G}_{I}(t)$ e $\widetilde{G}_{Q}(t)$ são processos Gaussianos mutuamente independentes que possuem a mesma função covariância. Apesar da análise feita no presente trabalho poder ser aplicada a processos Gaussianos com diferentes funções covariância $C(\tau)$, adotou-se o modelo de correlação exponencial [12] para $C(\tau)$ :

$$
C(\tau)=\mathbf{E}\left\{\left[\widetilde{G}^{*}(t)\right][\widetilde{G}(t+\tau)]\right\}=e^{-2 \pi B_{d} \tau}
$$

em que $B_{d}$ é a banda Doppler. Para um instante de tempo fixo, $t=k T$, a envoltória do desvanecimento $A_{k} \triangleq$ $\sqrt{\widetilde{G}_{I}^{2}(k T)+\jmath \widetilde{G}_{Q}^{2}(k T)}$ (onde $1 / T$ é a taxa de sinalização) tem função densidade de probabilidade Rayleigh dada por [7]:

$$
f_{A}(a)=\left\{\begin{array}{lll}
2 a e^{-a^{2}}, & \text { se } & a>0 \\
0, & \text { caso } & \text { contrário. }
\end{array}\right.
$$

O canal discreto mostrado na Fig. 2 será denominado canal discreto com correlação exponencial (CDCE). O sinal na entrada do quantizador no $k$-ésimo intervalo é dado por $R_{k}=$ $A_{k} S_{k}+N_{k}$, em que $S_{k}=\left(2 X_{k}-1\right) E_{s}, E_{s}$ é a energia do sinal transmitido, $A_{k}$ é uma variável aleatória Rayleigh e $N_{k}$ é uma variável aleatória Gaussiana com variância $N_{0} / 2$. No sistema considerado, um quantizador escalar uniforme é utilizado para mapear $R_{k}$ em $Y_{k}$ da seguinte forma:

$$
Y_{k}=j \quad \text { se } \quad R_{k} \in R_{D_{j}}, \quad j=0,1,2
$$

em que as regiões de decisão $R_{D_{j}}$ são definidas por:

$$
\begin{aligned}
& R_{D_{0}}=\{r \in R: r<-\Delta\} \\
& R_{D_{1}}=\{r \in R:-\Delta<r<\Delta\} \\
& R_{D_{2}}=\{r \in R: r>\Delta\}
\end{aligned}
$$

onde $\Delta$ é o passo do quantizador. A Fig. 3 ilustra o referido mapeamento. Define-se o passo do quantizador normalizado por $\delta=\Delta / \sqrt{E_{s}}$. Seja $q_{i, j}\left(a_{k}\right)=P\left(Y_{k}=j \mid X_{k}=i, A_{k}=\right.$ $a_{k}$ ) uma probabilidade condicional. Considerando-se $i=0$, tem-se para as regiões de decisão da Fig. 3 que:

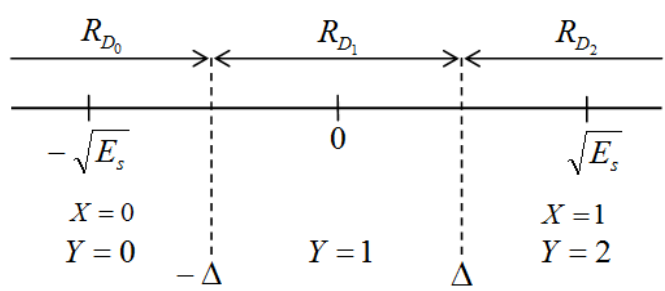

Fig. 3. Regiões de decisão para um canal discreto com 3 níveis de quantização.

$$
\begin{aligned}
& q_{0,0}\left(a_{k}\right)=Q\left(\sqrt{\frac{2 E_{s}}{N_{0}}}\left(\delta-a_{k}\right)\right) \\
& q_{0,1}\left(a_{k}\right)=Q\left(\sqrt{\frac{2 E_{s}}{N_{0}}}\left(a_{k}-\delta\right)\right)-Q\left(\sqrt{\frac{2 E_{s}}{N_{0}}}\left(a_{k}+\delta\right)\right) \\
& q_{0,2}\left(a_{k}\right)=Q\left(\sqrt{\frac{2 E_{s}}{N_{0}}}\left(\delta+a_{k}\right)\right)
\end{aligned}
$$

Pela simetria da constelação e das regiões de decisão, obtém-se $q_{0, j}\left(a_{k}\right)=q_{1,2-j}\left(a_{k}\right)$. Define-se, $q_{0, j} \triangleq P\left(Y_{k}=j \mid X_{k}=i\right)$. Então

$$
q_{1,2-j}=q_{0, j}=\mathbf{E}_{A_{k}}\left[q_{0, j}\left(A_{k}\right)\right]=\int_{0}^{\infty} q_{0, j}(a) 2 a e^{-a^{2}} d a .
$$

Define-se o processo de ruído ternário $\left\{Z_{k}\right\}_{k=1}^{\infty}$ em que $Y_{k}=$ $2 X_{k}+(-1)^{X_{k}} Z_{k}$, ou seja,

$$
Y_{k}=\left\{\begin{array}{rll}
Z_{k}, & \text { se } & X_{k}=0 \\
2-Z_{k}, & \text { se } & X_{k}=1
\end{array}\right.
$$

Assim, se $Z_{k}=0$, o canal tem boa qualidade e o sinal transmitido é recebido corretamente. Se $Z_{k}=1$, o canal apresenta distorções, gerando um apagamento na recepção. Se $Z_{k}=2$, ocorre um erro na recepção. Em geral, o canal discreto pode ser especificado pela probabilidade condicional:

$$
\begin{aligned}
& P\left(Y_{1}=y_{1}, \ldots, Y_{n}=y_{n} \mid X_{1}=x_{1}, \ldots, X_{n}=x_{n}\right) \\
& =P\left(Z_{1}=\frac{y_{1}-2 x_{1}}{(-1)^{x_{1}}}, \ldots, Z_{n}=\frac{y_{n}-2 x_{n}}{(-1)^{x_{n}}}\right)
\end{aligned}
$$

onde

$$
P\left(Z_{1}=z_{1}, \ldots, Z_{n}=z_{n}\right)=\mathbf{E}_{A_{1} A_{2} \ldots A_{n}}\left[\prod_{k=1}^{n} q_{0, z_{k}}\left(A_{k}\right)\right] .
$$

Para $n=1$, uma expressão fechada para $P_{\mathrm{CDCE}}\left(Z_{k}=j\right)$ para um canal com desvanecimento Rayleigh é dada por [13]:

$$
\begin{aligned}
& P_{\mathrm{CDCE}}(2)=m(-\delta) \\
& P_{\mathrm{CDCE}}(1)=m(\delta)-m(-\delta) \\
& P_{\mathrm{CDCE}}(0)=1-m(\delta)
\end{aligned}
$$

onde

$m(\delta)=1-Q(\delta \sqrt{2 \varphi})-\frac{1}{\sqrt{\frac{1}{\varphi}+1}}\left[1-Q\left(\frac{\delta \sqrt{2 \varphi}}{\sqrt{\frac{1}{\varphi}+1}}\right)\right] e^{\frac{-\delta^{2}}{\left(\frac{1}{\varphi}+1\right)}}$ 
e $\varphi=E_{s} / N_{0}$. Para calcular a probabilidade de uma seqüência de ruídos de comprimento 2 e 3 para o CDCE, será utilizado (14), como mostrado a seguir para $n=2$

$P_{\mathrm{CDCE}}\left(z_{1} z_{2}\right)=\int_{0}^{\infty} \int_{0}^{\infty} q_{0, z_{1}}\left(a_{1}\right) q_{0, z_{2}}\left(a_{2}\right) p_{A_{1} A_{2}}\left(a_{1}, a_{2}\right) d a_{1} d a_{2}$

onde as expressões para $q_{0, z_{i}}\left(a_{k}\right)$ são definidas em (10)(12) e a função densidade de probabilidade conjunta de duas variáveis aleatórias $A_{1}$ e $A_{2}$ para um canal com desvanecimento Rayleigh é dada por [14]

$$
p_{A_{1} A_{2}}\left(a_{1}, a_{2}\right)=\frac{4 a_{1} a_{2}}{1-\rho^{2}} e^{-\left(a_{1}^{2}+a_{2}^{2}\right) /\left(1-\rho^{2}\right)} I_{0}\left(\frac{2 \rho a_{1} a_{2}}{1-\rho^{2}}\right)
$$

onde $\rho=e^{-2 \pi B_{d} T}$ e $I_{0}(x)$ é a função de Bessel modificada de primeira espécie e ordem zero. A função densidade de probabilidade conjunta de três variáveis aleatórias $A_{1}, A_{2}$ e $A_{3}$ para um canal com desvanecimento Rayleigh é dada por [14]

$$
\begin{aligned}
p_{A_{1} A_{2} A_{3}}\left(a_{1}, a_{2}, a_{3}\right) & =\frac{8 a_{1} a_{2} a_{3}}{\left(\rho^{2}-1\right)^{2}} e^{-\frac{\left(a_{1}^{2}+a_{3}^{2}\right)}{1-\rho^{2}}-\frac{a_{2}^{2}\left(\rho^{2}+1\right)}{1-\rho^{2}}} \\
& \times I_{0}\left(\left|\frac{2 a_{1} a_{2} \rho}{\left(1-\rho^{2}\right)}\right|\right) I_{0}\left(\left|\frac{2 a_{2} a_{3} \rho}{\left(1-\rho^{2}\right)}\right|\right) .
\end{aligned}
$$

Logo

$$
\begin{aligned}
P_{\mathrm{CDCE}}\left(z_{1} z_{2} z_{3}\right)= & \int_{0}^{\infty} \int_{0}^{\infty} \int_{0}^{\infty} q_{0, z_{1}}\left(a_{1}\right) q_{0, z_{2}}\left(a_{2}\right) q_{0, z_{3}}\left(a_{3}\right) \\
& \times p_{A_{1} A_{2} A_{3}}\left(a_{1}, a_{1}, a_{3}\right) d a_{1} d a_{2} d a_{3}
\end{aligned}
$$

As probabilidades de seqüências de ruído de comprimento 3 do CDCE, dadas em (19), serão usadas nas próximas seções para calcular os parâmetros do MA, de modo que este seja uma boa aproximação do CDCE. O valor do passo de quantização será determinado de modo a maximizar a capacidade do canal CDCE. Para este fim, será analisado na próxima seção a capacidade deste canal.

\section{CAPACIDADE DO CANAL}

Seja o canal de comunicação ergódico e estacionário, com alfabeto de entrada $\{0,1\}$, alfabeto de saída $\{0,1,2\}$ e com processo de saída descrito por:

$$
Y^{n}=2 X^{n}+(-1)^{X^{n}} Z^{n}
$$

em que $X^{n}=\left\{X_{0}, X_{1}, \ldots, X_{n-1}\right\} \in\{0,1\}^{n}$ e $Y^{n}=$ $\left\{Y_{0}, Y_{1}, \ldots, Y_{n-1}\right\}, Z^{n}=\left\{Z_{0}, Z_{1}, \ldots, Z_{n-1}\right\} \in\{0,1,2\}^{n}$, sendo $n$ o número de utilizações do canal. O processo de saída $Y^{n}$ é independente do processo de entrada $X^{n}$ e as operações matemáticas em (20) são realizadas termo a termo. A capacidade desse canal é dada por [15]

$$
C=\lim _{n \rightarrow \infty} \max _{p\left(x^{n}\right)} \frac{1}{n} I\left(X^{n} ; Y^{n}\right),
$$

onde

$$
I\left(X^{n} ; Y^{n}\right)=H\left(Y^{n}\right)-H\left(Z^{n}\right)
$$

é a informação mútua entre $X^{n}$ e $Y^{n}$. Define-se $C^{(n)}$ como

$$
\begin{aligned}
C^{(n)} & =\max _{p\left(x^{n}\right)} \frac{1}{n} I\left(X^{n} ; Y^{n}\right) \\
& =\frac{1}{n}\left(\widetilde{H}\left(Y^{n}\right)-H\left(Z^{n}\right)\right)
\end{aligned}
$$

onde $\widetilde{H}\left(Y^{n}\right)$ é a máxima entropia de $Y^{n}$ e $H\left(Z^{n}\right)$ é a entropia de $Z^{n}$. Para calcular $C^{(n)}$ é preciso achar a distribuição de entrada que maximiza $H\left(Y^{n}\right)$. O cálculo de $C^{(n)}$ para $n=$ 1,2 será demonstrado a seguir. Seja $\mathbf{T}^{(n)}=\left[t_{\mathbf{i}, \mathbf{j}}^{(n)}\right]$ a matriz de transição do canal, onde

$$
t_{\mathbf{i}, \mathbf{j}}^{(n)}=P\left(Y^{n}=\mathbf{j} \mid X^{n}=\mathbf{i}\right), \mathbf{j} \in Y^{n}, \mathbf{i} \in X^{n} .
$$

Considerando-se $G_{i}=P(Z=i), i \in\{0,1,2\}$, a matriz $\mathbf{T}^{(1)}$ é

$$
\mathbf{T}^{(1)}=\left[\begin{array}{ccc}
G_{0} & G_{1} & G_{2} \\
G_{2} & G_{1} & G_{0}
\end{array}\right]
$$

A matriz $\mathbf{T}^{(1)}$ pode ser dividida em submatrizes $\mathbf{Q}_{1}$ e $\mathbf{Q}_{2}$

$$
\mathbf{Q}_{1}=\left[\begin{array}{cc}
G_{0} & G_{2} \\
G_{2} & G_{0}
\end{array}\right], \mathbf{Q}_{2}=\left[\begin{array}{c}
G_{1} \\
G_{1}
\end{array}\right] .
$$

Pode ser observado que estas submatrizes representam canais fracamente simétricos [16], em que as linhas são permutáveis entre si e a soma dos elementos de cada coluna é igual. Para estes canais, a capacidade é obtida para uma distribuição de entrada uniforme [16]. Assim, obtém-se:

$$
\begin{aligned}
C^{(1)} & =\widetilde{H}\left(Y^{1}\right)-H\left(Z^{1}\right) \\
& =1-\left(G_{0}+G_{2}\right) \log \left(G_{0}+G_{2}\right)+G_{0} \log \left(G_{0}\right) \\
& +G_{2} \log \left(G_{2}\right)
\end{aligned}
$$

onde $G_{i}$ para o CDCE é dado em (15)-(17). A matriz $\mathbf{T}^{(2)}$ também pode ser dividida em quatro submatrizes. A capacidade $C^{(2)}$ é obtida para de uma distribuição de entrada uniforme, dado que estas submatrizes representam canais fracamente simétricos. Seja $G_{i, j}=P\left(Z_{k}=i, Z_{k+1}=j\right)$, então

$$
\begin{aligned}
C^{(2)} & =\frac{1}{2}\left(\widetilde{H}\left(Y^{2}\right)-H\left(Z^{2}\right)\right) \\
\frac{1}{2}[- & \left(G_{00}+G_{02}+G_{20}+G_{22}\right) \log \left(\frac{G_{00}+G_{02}+G_{20}+G_{22}}{4}\right) \\
& -G_{11} \log \left(G_{11}\right)-\left(G_{01}+G_{21}\right) \log \left(\frac{G_{01}+G_{21}}{2}\right) \\
& -\left(G_{10}+G_{12}\right) \log \left(\frac{G_{10}+G_{12}}{2}\right)+G_{00} \log \left(G_{00}\right) \\
& +G_{01} \log \left(G_{01}\right)+G_{02} \log \left(G_{02}\right)+G_{10} \log \left(G_{10}\right) \\
& +G_{11} \log \left(G_{11}\right)+G_{12} \log \left(G_{12}\right)+G_{20} \log \left(G_{20}\right) \\
& \left.+G_{21} \log \left(g_{21}\right)+G_{22} \log \left(G_{22}\right)\right]
\end{aligned}
$$

onde os valores de $G_{i j}$ para o CDCE podem ser calculados usando-se (18). Neste trabalho, o valor de $\delta$ será selecionado de modo a maximizar o valor de $C^{(2)}$. A Fig. 4 mostra os gráficos da capacidade do canal, em bits/uso, versus $\delta$ para 
$E_{s} / N_{0}=5 \mathrm{~dB}$ e $B_{d} T=0,01$. Esta figura indica que o melhor valor de $\delta$ está em torno de 0,25 . A partir de outras curvas traçadas para diversos valores de $B_{d} T$ e $E_{s} / N_{0}$, é possível concluir que o valor de $\delta$ que maximiza a capacidade do canal diminui com o aumento de $E_{s} / N_{0}$. Alguns valores podem ser vistos na Tabela I para o caso em que $B_{d} T=0,01$. Outra observação que pode ser feita é que os valores de $\delta$ que maximizam a capacidade do canal não variam com $B_{d} T$.

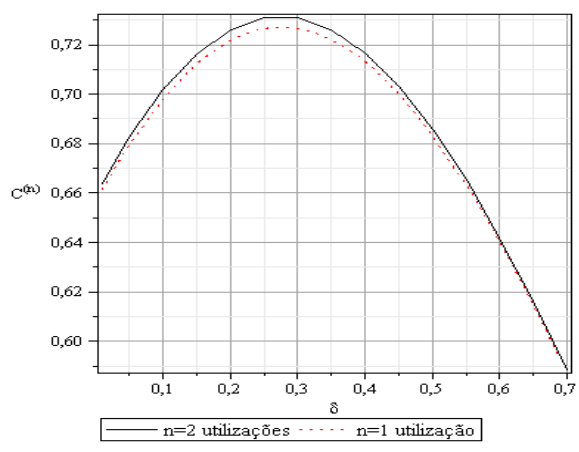

Fig. 4. $C^{(1)}, C^{(2)}$ versus $\delta$ para o CDCE com $B_{d} T=0,01$ e $E_{s} / N_{0}=5$ $\mathrm{dB}$

TABELA I

VALORES Ótimos de $\delta$ EM FUnÇÃo DE $E_{s} / N_{0}$ PARA $B_{d} T=0,01$.

\begin{tabular}{|c|c|}
\hline$E_{s} / N_{0}$ & $\delta$ \\
\hline $0 \mathrm{~dB}$ & 0,45 \\
\hline $2 \mathrm{~dB}$ & 0,35 \\
\hline $5 \mathrm{~dB}$ & 0,25 \\
\hline $8 \mathrm{~dB}$ & 0,20 \\
\hline $10 \mathrm{~dB}$ & 0,15 \\
\hline
\end{tabular}

\section{ESTIMAÇÃO DOS PARÂMETROS DO MA}

Empregou-se nesta seção o método de minimização da divergência para calcular os parâmetros do MA, para que este seja uma boa aproximação do CDCE, o qual é descrito por três parâmetros: a relação sinal ruído $E_{s} / N_{0}$, a banda Doppler normalizada $B_{d} T$ e o passo do quantizador $\delta$. É possível estimar os cinco parâmetros do MA, $\alpha, \beta, \xi, q \mathrm{e}$ $Q$, utilizando-se a minimização da divergência, mensurada pela distância Kullback-Leibler [10], com a restrição que o CDCE e o MA tenham o mesmo coeficiente de correlação, a mesma probabilidade de ocorrer uma recepção correta e a mesma probabilidade de ocorrer um apagamento, isto é, $\operatorname{Cor}_{\mathrm{CDCE}}=\mathrm{Cor}_{\mathrm{MA}}, P_{\mathrm{CDCE}}(0)=P_{\mathrm{MA}}(0)$ e $P_{\mathrm{CDCE}}(1)=P_{\mathrm{MA}}(1)$. A divergência é expressa por:

$$
\lim _{n \rightarrow \infty} \frac{1}{n} D_{n}\left(P_{\mathrm{CDCE}} \| P_{\mathrm{MA}}\right)
$$

onde $\frac{1}{n} D_{n}\left(P_{\mathrm{CDCE}} \| P_{\mathrm{MA}}\right)$ é a distância normalizada de $n$-ésima ordem entre as distribuições do CDCE e do MA, em que $D_{n}\left(P_{\mathrm{CDCE}} \| P_{\mathrm{MA}}\right)$ é definida por:

$$
D_{n}\left(P_{\mathrm{CDCE}} \| P_{\mathrm{MA}}\right)=\sum_{z^{n} \in\{0,1,2\}^{n}} P_{\mathrm{CDCE}}\left(z^{n}\right) \log _{2} \frac{P_{\mathrm{CDCE}}\left(z^{n}\right)}{P_{\mathrm{MA}}\left(z^{n}\right)}
$$

onde $P_{\mathrm{CDCE}}\left(z^{n}\right)$ é dado por (14) e $P_{\mathrm{MA}}\left(z^{n}\right)$ é calculado matricialmente por (1), utilizando-se as matrizes $\mathbf{P}(0), \mathbf{P}(1)$ e $\mathbf{P}(2)$, dadas em (2)-(4). A Tabela II mostra os parâmetros obtidos com a minimização de $D_{3}\left(P_{\mathrm{CDCE}} \mid P_{\mathrm{MA}}\right)$ para valores relevantes dos parâmetros do CDCE.

Para mensurar a exatidão do modelo MA em aproximar o CDCE, será feita uma comparação entre suas funções autocorrelação. A utilização da função autocorrelação para testar a exatidão de um modelo foi proposta em [17] e é largamente utilizada na literatura [7], [18], [19]. A função autocorrelação do CDCE é dada por:

$$
\begin{aligned}
R(m) & =\mathbf{E}\left[Z_{i}, Z_{i+m}\right] \\
& =1 \cdot 1 \cdot P\left(Z_{i}=1, Z_{i+m}=1\right)+1 \cdot 2 \cdot P\left(Z_{i}=1, Z_{i+m}=2\right) \\
& +2 \cdot 1 \cdot P\left(Z_{i}=2, Z_{i+m}=1\right)+2 \cdot 2 \cdot P\left(Z_{i}=2, Z_{i+m}=2\right)
\end{aligned}
$$

onde $P\left(Z_{i}=z_{i}, Z_{i+m}=z_{i+m}\right)$ é calculado usando-se (18).

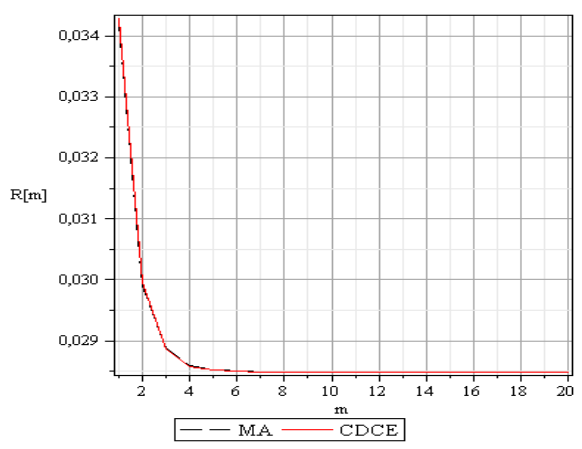

Fig. 5. Comparação das funções autocorrelação do CDCE e do MA, para $B_{d} T=0,1, \delta=0,25$ e $E_{s} / N_{0}=5 \mathrm{~dB}$.

As curvas da função autocorrelação para 20 valores de $m$ do CDCE e do MA são comparadas na Fig. 5. Os parâmetros do modelo CDCE são $B_{d} T=0,1$ e $E_{s} / N_{0}=5 \mathrm{~dB}$. É possível notar que as curvas são praticamente idênticas, o que mostra que o MA possui um comportamento muito parecido com o CDCE para os parâmetros mencionados.

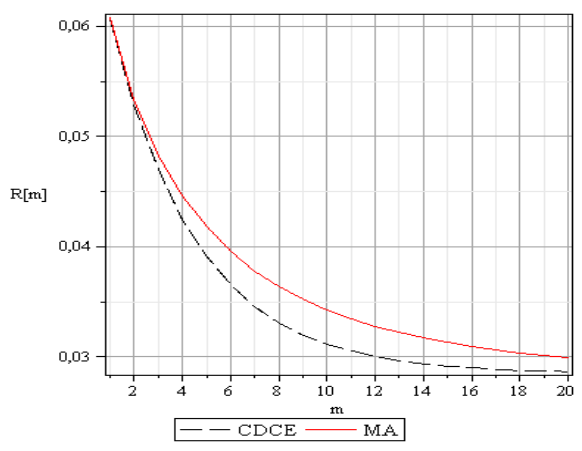

Fig. 6. Comparação das funções autocorrelação do CDCE e do MA, para $B_{d} T=0,01, \delta=0,25$ e $E_{s} / N_{0}=5 \mathrm{~dB}$.

A Fig. 6 também ilustra o comportamento da função autocorrelação para 20 valores de $m$ do CDCE e do MA, porém, para um CDCE com desvanecimento mais lento, com $B_{d} T=0,01$ e $E_{s} / N_{0}=5 \mathrm{~dB}$. Conclui-se que o MA 
TABELA II

VALORES DOS PARÂMETROS DO MA QUE MODELAM UM CDCE.

\begin{tabular}{|c|c|c|c|c|c|}
\hline Parâmetros do CDCE & $\alpha$ & $\beta$ & $\xi$ & $q$ & $Q$ \\
\hline$E_{s} / N_{0}=0 \mathrm{~dB}, B_{d} T=0,01, \delta=0,45$ & 0,3817 & 0,1001 & 0,1051 & 0,0866 & 0,1003 \\
\hline$E_{s} / N_{0}=2 \mathrm{~dB}, B_{d} T=0,01, \delta=0,35$ & 0,3554 & 0,0901 & 0,0701 & 0,1024 & 0,0795 \\
\hline$E_{s} / N_{0}=5 \mathrm{~dB}, B_{d} T=0,01, \delta=0,25$ & 0,3374 & 0,0751 & 0,0351 & 0,1711 & 0,0717 \\
\hline$E_{s} / N_{0}=8 \mathrm{~dB}, B_{d} T=0,01, \delta=0,20$ & 0,3247 & 0,0501 & 0,0201 & 0,1915 & 0,0488 \\
\hline$E_{s} / N_{0}=10 \mathrm{~dB}, B_{d} T=0,01, \delta=0,15$ & 0,4430 & 0,0151 & 0,0151 & 0,2580 & 0,0580 \\
\hline
\end{tabular}

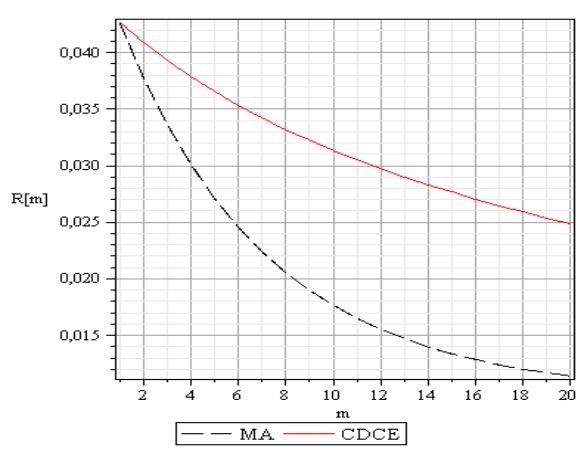

Fig. 7. Comparação das funções autocorrelação do CDCE e do MA, para $B_{d} T=0,001, \delta=0,15$ e $E_{s} / N_{0}=10 \mathrm{~dB}$.

aproxima razoavelmente o CDCE. A Fig. 7 mostra a mesma comparação feita anteriormente entre a função autocorrelação do CDCE e do MA, porém para um CDCE com desvanecimento ainda mais lento, com $B_{d} T=0,001$ e $E_{s} / N_{0}=10 \mathrm{~dB}$. É fácil perceber a grande divergência das curvas. Isso decorre do fato de que para obter-se uma modelagem precisa do CDCE com $B_{d} T \leq 10^{-3}$, deve-se utilizar $P_{\mathrm{CDCE}}\left(z^{n}\right)$ com $n>3$. Expressões teóricas para obtenção destas probabilidades não são conhecidas na literatura, entretanto, simulações para a obtenção destas podem ser empregadas. Os resultados desta seção mostram que o modelo MA proposto para o canal CDCE é preciso para $B_{d} T \geq 0,01$ e para uma ampla faixa de $E_{s} / N_{0}$ (curvas para diversos valores de $E_{s} / N_{0}$ não são mostradas devido à limitação de espaço).

\section{Conclusões}

Este trabalho propôs um modelo FSMC não binário, com três níveis de quantização, denominado de modelo com apagamento e denotado por MA. Este foi idealizado para que haja uma menor perda de informação, quando comparado a FSMC binários, com apenas dois níveis de quantização. Foram descritas as matrizes de distribuição de erros, acertos e de apagamentos, bem como a matriz de transição de estados, em função dos parâmetros $\alpha, \beta, \xi, q$ e $Q$ do MA. Também foram desenvolvidas as estatísticas do canal discreto com correlação exponencial (CDCE), o qual desejase modelar com o MA. O valor do passo do quantizador $\delta$ foi determinado através dos cálculos da capacidade do canal MA para 1 e 2 utilizações, para diferentes valores dos parâmetros do CDCE, $B_{d} T$ e $E_{s} / N_{0}$. Sabendo-se o passo adequado do quantizador, foi possível obter as probabilidades de decodificação sem sucesso do CDCE, as quais foram utilizadas para determinação dos parâmetros do MA através da minimização da distância Kullback-Leibler. A validação do modelo foi realizada comparando-se a função autocorrelação do MA e do CDCE.

\section{REFERNCIAS}

[1] L. N. Kanal and A. R. K. Sastry, "Models for channels with memory and their applications to error control," Proc. of the IEEE, vol. 66, no. 7, pp. 724-744, July 1978.

[2] M. Mushkin and I. Bar-David, "Capacity and coding for the GilbertElliot channel," IEEE Trans. Inf. Theory, vol. 35, no. 6, pp. 1277-1290, Nov. 1989.

[3] A. W. Eckford, F. R. Kschischang, and S. Pasupathy, "Analysis of lowdensity parity check codes for the Gilbert-Elliott channels," IEEE Trans. Inf. Theory, vol. 51, pp. 3872-3889, Nov. 2005.

[4] L. Wilhelmsson and L. B. Milstein, "On the effect of imperfect interleaving for the Gilbert-Elliott channel," IEEE Trans. Commun., vol. 47, pp. 681-688, May 1999.

[5] H. Turin and R. van Nobelen, "Hidden Markov modeling of flat fading channels," IEEE J. Select Areas Commun., vol. 16, pp. 1809-1817, Dec. 1998.

[6] J. Yee and E. Weldon, "Evaluation of the performance of error-correcting codes on a Gilbert channel," IEEE Trans. Commun., vol. 43, pp. 23162323, Aug. 1995.

[7] C. Pimentel, T. H. Falk, and L. Lisbôa, "Finite-state Markov modeling of correlated Rician fading channels," IEEE Trans. Veh. Technol., vol. 53, no. 5, pp. 1491-1501, Sep. 2004.

[8] N. Phamdo and F. Alajaji, "Soft-decision demodulation design for COVQ over white, colored and ISI Gaussian channels," IEEE Trans. Commun., vol. 48, pp. 1499-1506, Sep. 2000.

[9] J. Singh, O. Dabeer, and U. Madhow, "Transceiver design with lowprecision analog-to-digital conversion: An information-theoretic perspective," 2008, submitted to IEEE Trans. Inf. Theory. Preprint avaiable at arXiv:0804.1172v1.

[10] L. Zhong, F. Alajaji, and G. Takahara, "A binary communication channel with memory based on a finite queue," IEEE Trans. Inf. Theory, vol. 53, no. 8, pp. 2815-2840, Aug. 2007.

[11] C. Pimentel and V. C. da Rocha Jr., "On the power spectral density of constrained sequences," IEEE Trans. Commun., vol. 55, no. 3, pp. 409-416, Mar. 2007.

[12] Y. Chen and C. Tellambura, "Infinite series representation of the trivariate and quadrivariate Rayleigh distribution and their applications," IEEE Trans. Commun., vol. 53, no. 12, pp. 2092-2101, Dec. 2005.

[13] F. Alajaji and N. C. Phamdo, "Soft-decision COVQ for Rayleigh-fading channels," IEEE Commun. Letters, vol. 2, no. 6, pp. 162-164, June 1998.

[14] K. S. Miller, "Complex Gaussian processes," SIAM Review, vol. 11, no. 4, pp. 544-567, Oct. 1969.

[15] R. G. Gallager, Information Theory and Reliable Communications. New York: Wiley, 1968.

[16] T. Cover and J. Thomas, Elements of Information Theory, 2nd ed. Wiley-Interscience, 2006.

[17] C. Tan and N. C. Beaulieu, "On first-order Markov modeling for the Rayleigh fading channel," IEEE Trans. Commun., vol. 48, pp. 2032 2040, Dec. 2000.

[18] L. Zhong, F. Alajaji, and G. Takahara, "A model for correlated Rician fading channels based on a finite queue," IEEE Trans. Veh. Technol., vol. 57, no. 1, pp. 79-89, Jan. 2008.

[19] W. Kumwilaisak, C. Kuo, and D. Wu, "Fading channel modeling via variable-length Markov chain technique," IEEE Trans. Veh. Technol., vol. 57, pp. 1338-1358, May 2008. 